\title{
The Analysis of Light Coupling and Propagation for a Composite Fiber-Dielectric Slab with a Conductor Cladding
}

\author{
Kwang-Hee Kwon*, Jae-Won Song, and Jeong-Hoon Kim \\ Kyungpook National University, Daegu 702-701, KOREA \\ Euy-Don Park and Seok-Woo Son \\ LG Ltd. Gyeongbuk 730-360, KOREA
}

(Received November 22, 2002)

\begin{abstract}
A theoretical presentation of evanescent coupling is offered with respect to the refractive indexes between a side-polished optical fiber and an infinitely planar waveguide with a conductor cladding (PWGCC). The PWG is suspended at a constant distance from an unclad fiber core and attached with the perfect conductor (PEC) on one side. The behavior of the distributed coupler is examined using a coupled mode model, which takes account of the two dimensions of the waveguide configuration. The coupling and propagation of light were found to depend on both the relationship between the refractive index values of each structure and the configuration of the side-polished fiber used in the PWGCC. The spreading of light in the unconfined direction of the PWGCC is described in terms of a simple geometrical interpretation of the synchronization condition that is in agreement with a previous investigation of the problem based on the coupled-mode theory (CMT). The power of the light propagation in the fiber decreased exponentially along the fiber axis as it was transferred to the PWGCC, where it was carried away.
\end{abstract}

OCIS code : 250.0250 .

\section{INTRODUCTION}

The exchange of energy between an optical fiber and a PWG placed alongside in close proximity has attracted much attention over the years due to its challenging intrinsic complexity and potential application in many fields. A side-polished fiber-slab waveguide structure, consisting of a single-mode optical Dshaped fiber with a flat cladding surface attached to the PWG overlay, has also been used in channeldropping filters [1], polarizers [2], intensity modulators [3,4] and sensors [5]. In particular, polarizers have been investigated using the coupling between a side-polished fiber and a dielectric slab with a metal film on one side. For example, Marcuse [6] and Shu Zheng [7] developed a theoretical analysis using CMT as a single-mode fiber-optic polished coupler with the PWG layer formed by optical glue between the two half-blocks of the coupler. However, the coupling between a fiber and a PWGCC has not yet been investigated. Therefore, the current study investigated a composite fiber-slab structure with a conducting surface attached to one side of the PWG, thereby asymmetrically extending the analysis by Shu Zheng [7]. It was assumed that the combined system of a fiber and PWGCC could also be regarded as a ridge waveguide, as in references [6], [7] and [8]. According to these references, when the fiber mode is essentially synchronous with the ridge mode, considerable beating occurs initially between the guided and unguided compound modes of the system until a guided ridge mode is established through the PWGCC. When the fiber mode is infinitely coupled, many of the PWGCC modes suffer coupling-induced attenuation. Plus, when the fiber propagation constant lies deep within the range of the propagation constants of the continuum slab modes in the PWGCC, an exponential decay of the light carried in the fiber is observed. Accordingly, to represent the effect of coupling between the two structures, the fiber-slab system was described using coupled-mode equations obtained by expanding the total field as a superposition of the slab mode and 


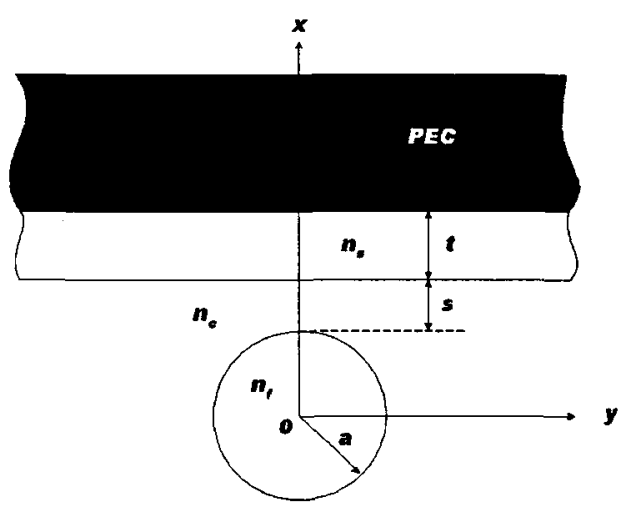

FIG. 1. Cross-sectional view of the planar wave guide under the perfect conductor(PWGCC) suspended above fiber.

fiber modes, even though these modes are not mutually orthogonal, and then representing the amplitude of the electric field in the propagating direction using numerical techniques.

\section{COUPLED MODE EQUATIONS}

Longitudinal and cross-sectional views of the distributed single-mode fiber-to PWGCC coupler are presented in Fig. 1. The guided modes of the fiber and PWGCC were used to describe the behavior of the light propagation in the combined system. Scalar approximation was applied whenever all the refractive index differences remain small [9]. Therefore, based on scalar approximation the scalar wave equation is as follows:

$$
\nabla_{t}^{2} \mathbf{E}+\frac{\partial^{2} \mathbf{E}}{\partial z^{2}}+k^{2} n^{2}(x, y) \mathbf{E}=0
$$

where $\mathbf{E}$ is the transverse electric field of the fiberPWGCC system, $\nabla_{t}^{2}=\frac{\partial^{2}}{\partial x^{2}}+\frac{\partial^{2}}{\partial y^{2}}$ and $n(x, y)$ defines the refractive index profile throughout the transverse $(x, y)$ plane. As shown in Fig. 1, the index profiles of the unperturbed fiber and PWGCC are

$$
n_{f}(x, y)=\left\{\begin{array}{l}
n_{f} \text { for } x^{2}+y^{2} \leq a^{2} \\
n_{c} \text { for } x^{2}+y^{2}>a^{2}
\end{array}\right\}
$$

$$
n_{s}(x, y)=\left\{\begin{array}{ll}
n_{c} & \text { for } x<a+s \\
n_{s} & \text { for } a+s \leq x<a+s+t
\end{array}\right\}
$$

Therefore

$$
n^{2}(x, y)=n_{f}^{2}(x, y)+n_{s}^{2}(x, y)-n_{c}^{2}
$$

Where $n_{f}, n_{c}$ and $n_{s}$ are the refractive indices of the fiber, clad, and PWGCC, respectively. The transverse electric field in the perturbed or coupled fiber and PWGCC can be represented by the superposition of the guided-modes of the fiber $F_{n}$ and PWGCC $T_{n}(n=0,1,2, \cdots, K)$

$$
E(x, y, z)=\sum_{n}\left\{a_{n}(z) F_{n}(x, y) e^{-j \beta_{f n} z}+b_{n}(z) T_{n}(x, y) e^{-j \beta_{f n} z}\right\}
$$

Where $\left\{a_{0}(z), b_{n}(z)\right\}(n=0,1,2, K)$ are a set of $z$ dependent mode excitation coefficients. The coupled wave equations are obtained using the wave equations and the property of orthogonality for each transverse function, $F_{n}, T_{n}$. In the procedure of coupled wave equations, second derivatives of $a_{n}(z)$ and $b_{n}(z)$ are also neglected relative to $k^{2}$ since it is assumed that the field amplitudes only change slowly over the distance of one wavelength. The normalized mode function of the single-mode fiber ( $L P_{01}$ mode) has the coordinate system form shown in Fig. 1.

$$
F_{0}=N_{f} \begin{cases}\frac{J_{0}\left(k_{f} r\right)}{J_{0}\left(k_{f} a\right)} & r \leq a \\ \frac{K_{0}\left(\gamma_{f} r\right)}{K_{0}\left(\gamma_{f} a\right)} r>a\end{cases}
$$

Where $a$ is the fiber radius, $V_{f}=k a\left(\sqrt{n_{f}^{2}-n_{c}^{2}}\right)^{2}$ is the fiber-normalized frequency, $k_{f}$ and $\gamma_{f}$ are related to the propagation constant $\beta_{f 0}$ of the fiber based on the relation $k_{f}^{2}=n_{f}^{2} k^{2}-\beta_{f 0}^{2}$, and $\gamma_{f}^{2}=\beta_{f 0}^{2}-n_{c}^{2} k^{2}$. $N_{f}=\frac{\gamma_{f} J_{0}\left(k_{f} a\right)}{\sqrt{\pi} V_{f} J_{1}\left(k_{f} a\right)}$ is the normalization constant of the fiber mode. $J_{0}, J_{1}$, and $K_{0}$ are the Bessel and modified Bessel functions of the second kind, respectively. The fiber propagation constant $\beta_{f 0}$ is obtained from the $L P_{01}$-mode dispersion equation:

$$
k_{f} \frac{J_{1}\left(k_{f} a\right)}{J_{0}\left(k_{f} a\right)}=\gamma_{f} \frac{K_{1}\left(\gamma_{f} a\right)}{K_{0}\left(\gamma_{f} a\right)}
$$

The normalized PWGCC mode of the transverse order $n$ only has a field distribution in the case of $T E$ [10], which is satisfied by the boundary condition on the PEC. 


$$
S_{n}(x, y)=N_{s} \cos \left(\sigma_{n} y\right) \cdot\left\{\begin{array}{cl}
-e^{-\gamma_{s} t} \cdot \frac{\sin \left[k_{s}(x-a-s-t)\right]}{\sin \left(k_{s} t\right)} & \text { for } a+s<x \leq a+s+t \\
\exp \left[\gamma_{s}(x-a-s-t)\right] & \text { for } x \leq a+s
\end{array}\right.
$$

Where $t$ is the thickness of the PWGCC and $N_{s}=\frac{\sqrt{2} k_{s} t \cdot e^{\gamma_{s} t}}{V\left[D\left(t+a / \gamma_{s}\right)\right]^{1 / 2}}, n=0,1,2, \cdots, K$ is the normalization constant of the slab modes, while $k_{s}^{2}=$ $n_{s}^{2} k^{2}-\beta_{s}^{2}, \gamma_{c}^{2}=\beta_{s}^{2}-n_{c}^{2} k^{2}, \beta_{s n}^{2}=\beta_{s}^{2}-\sigma_{n}^{2}$, and $\sigma_{n}=\left(n+\frac{1}{2}\right) \frac{\pi}{D},(n=0,1,2, \cdots, K)$, are constants. The propagation constant $\beta_{s}$ is found from the eigenvalue equation of the PWGCC in the case of $T E[10]$ :

$$
-X \cdot \cot X=\sqrt{V_{s}^{2}-X^{2}}
$$

where $V_{s}^{2}=k^{2}\left(n_{s}^{2}-1\right) t^{2}, X=p t, p^{2}=n_{s}^{2} k^{2}-\beta_{s}^{2}$.
The minimum thickness $t$ required for a trapped wave in the case of $T E[10]$ is

$$
\frac{t}{\lambda_{0}}>\frac{1}{4\left(n_{s}^{2}-1\right)^{1 / 2}}
$$

The current study is only restricted to cases of a single $L P_{01}$ mode of guided fiber, so that $a_{m}=0$ for $m>0$. After multiplying and integrating with $F_{n}$ and $T_{n}$ over the transverse plane and using the orthogonality and wave equations, the CMT system of coupled equations for $a_{0}$ and $b_{m}$ is:

$$
\begin{gathered}
\frac{d a_{0}}{d z}=-\sum_{n}\left[B_{f 0 n} \frac{d b_{n}}{d z}+j K_{f 0 n} b_{n}\right] \cdot \exp \left\{j\left(\beta_{f 0}-\beta_{s n}\right) z\right\}-j Q_{f 00} a_{0} \\
\frac{d b_{m}}{d z}=-\sum_{n}\left[B_{s m 0} \frac{d b_{0}}{d z}+j K_{s m 0} a_{0}\right] \cdot \exp \left\{j\left(\beta_{s m}-\beta_{f 0}\right) z\right\}-j \sum_{n} Q_{s m n} b_{n} \cdot \exp \left\{j\left(\beta_{s m}-\beta_{s n}\right) z\right\}
\end{gathered}
$$

Among the predominant coupling coefficients resulting from the coupled-mode analysis in Eqs. (11) and (12), the four most relevant ones are the two crosscoupling coefficients $K$ and the two self-coupling coefficients $Q$ :

$$
\begin{aligned}
K_{f 0 n} & =\frac{k^{2}}{2 \beta_{f 0}} \int_{\text {area }}\left(n_{f}^{2}-n_{c}^{2}\right) F_{0} T_{n} d x d y \\
K_{s m 0} & =\frac{k^{2}}{2 \beta_{s m}} \int_{a r e a}\left(n_{s}^{2}-n_{c}^{2}\right) T_{m} F_{0} d x d y \\
Q_{f 00} & =\frac{k^{2}}{2 \beta_{f 0}} \int_{\text {area }}\left(n_{s}^{2}-n_{c}^{2}\right) F_{0} F_{0} d x d y \\
Q_{s m n} & =\frac{k^{2}}{2 \beta_{s m}} \int_{\text {area }}\left(n_{f}^{2}-n_{c}^{2}\right) T_{m} T_{n} d x d y
\end{aligned}
$$

The integrals in (13), (14), and (16) can be solved exactly, while for (15) an approximation is possible. Explicit expressions for these solutions are given in the appendix. $K_{f m n}$ and $K_{s m n}$ are cross-coupling coefficients that couple the fiber mode to the PWGCC modes and vice versa. The coefficients $Q_{f m n}$ and
$Q_{s m n}$ mediate self-coupling, yet the overlap integrals that appear are neglected due to the lack of orthogonality in the field functions $F_{m}$ and $T_{m}$ used in the expansion (5). The coupled Eqs. (11) and (12) are not exact because of the scalar approximation of Maxwell's equations, exclusion of the radiation modes, and neglect of the second derivatives of the expansion coefficients $a_{m}$ and $b_{m}$. To get modal solutions, constant wave amplitudes $A_{0}$ and $B_{m}$ are introduced via the relations

$$
\begin{aligned}
a_{0} & =A_{0} \cdot \exp \left\{j\left(\beta_{f 0}-\beta\right) z\right\}, \\
b_{m} & =B_{m} \cdot \exp \left\{j\left(\beta_{s m}-\beta\right) z\right\}
\end{aligned}
$$

Substitution of (17) into (11) and (12) yields the following equations that define the compound eigenmodes, Thus, the electric fields for the transverse direction can be represented using a compound mode equation for the propagating direction:

$$
\begin{aligned}
Q_{f 00} A_{0}+\sum_{n} K_{f 0 n} B_{n} & =\left(\beta-\beta_{f 0}\right) A_{0} \\
K_{s m 0} A_{0}+\sum_{n} Q_{s m a} B_{n} & =\left(\beta-\beta_{s m}\right) B_{m} \\
\text { for } m & =0,1,2, \cdots, K
\end{aligned}
$$


The Analysis of Light Coupling and Propagation for $\cdots-$ Kwang-Hee Kwo et al.

\section{NUMERICAL RESULTS AND DISCUSSION}

Numerical results are presented for the PWGCC that is a special asymmetric case in reference [7]. All the optical and structural parameters are defined as in reference [7], except for the PEC attached to the PWG. The investigation of the structure was carried out using a single-mode fiber (SMF) with the following parameters: light wavelength $\lambda=1.3 \mu \mathrm{m}$, fiber radius $a=2.5 \mu \mathrm{m}$, thickness of PWGCC $t=3 \mu \mathrm{m}$, refractive index of fiber cladding $n_{c}=1.46$, and refractive index of the PWGCC $n_{s}=1.4545$. The minimal distance

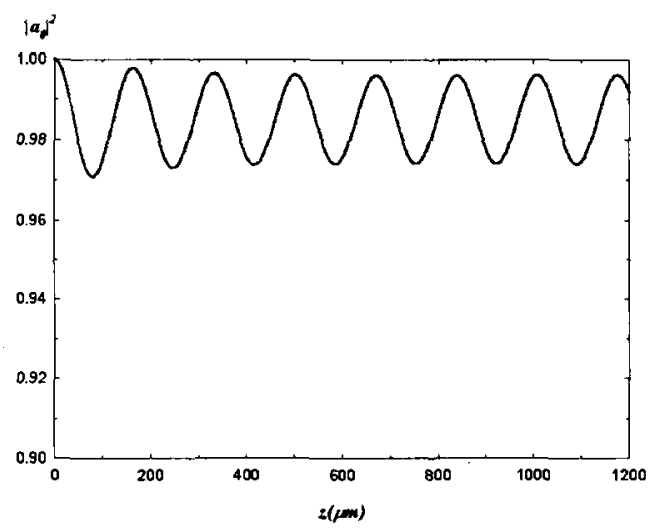

(a)

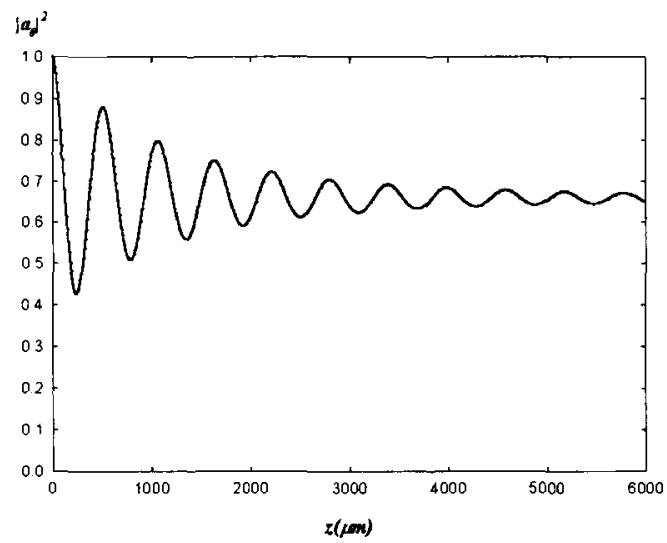

(c)

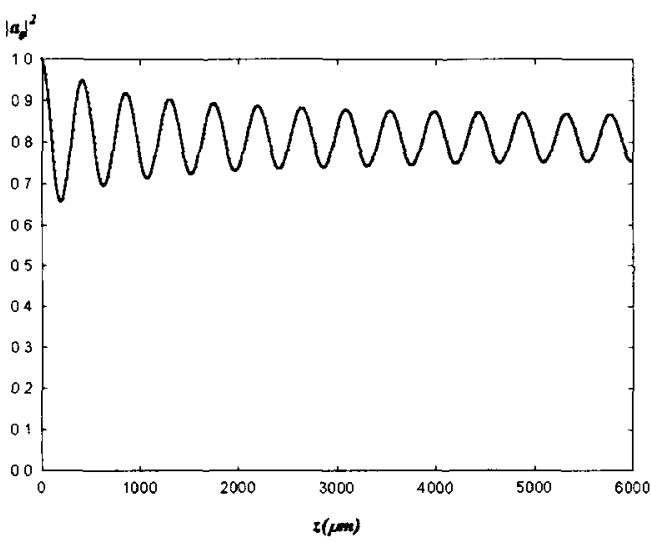

(b)

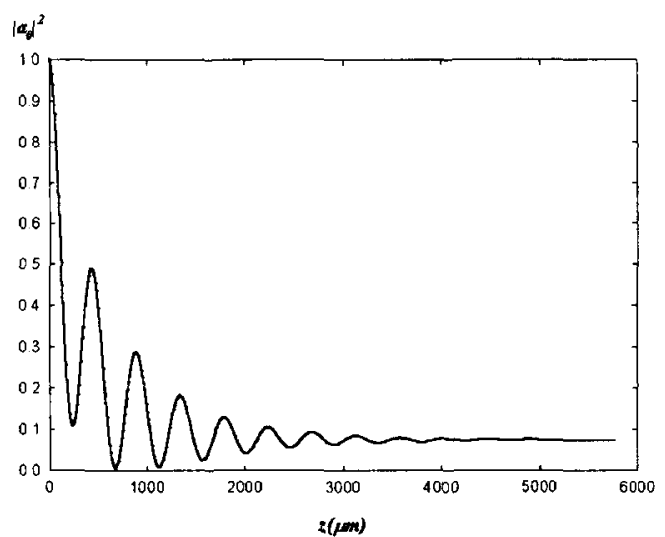

(d)

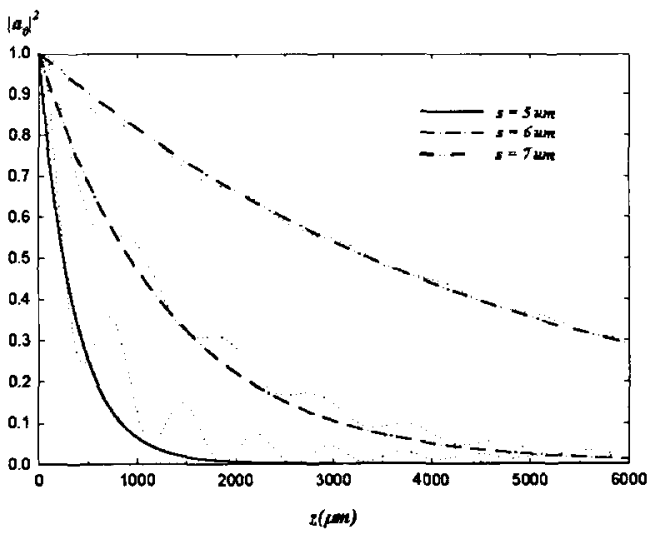

(e)

FIG. 2. Absolute squares of excitation coefficients of fiber mode as function of light propagation distance $z$ in fiber. The value of the refractive index for the fiber core was set at $n_{f}=$ (a) 1.4817 , (b) 1.4756 , (c) 1.4745 , and (d) 1.4709 , (e) fiber-mode loss to PWGCC, namely exponential curves for $s$. 
between the fiber core and the PWGCC is called the polishing depth and denoted by $s$. It was assumed that the thickness of the PWGCC satisfied the condition in (10) to propagate its guiding mode. The value of the refractive index of the fiber core $n_{f}$ varied around that of the PWGCC, i.e. $n_{f}>n_{s}, n_{f}=n_{s}$ and $n_{f}<n_{s}$, respectively. Then the propagation constant for the fiber $L P_{01}$ mode, $\beta_{f 0}$ was obtained using the solution of the eigenvalue equation for weakly guiding fibers [9], while the propagation constant for the PWGCC, $\beta_{s}$ was obtained using the Newton-Raphson method. To study the couple mode equation using the Runge-Kutta method, it was also assumed that only the $L P_{01}$ mode was initially launched through the fiber at $z=0$ starting at any $z=0$ where the coupling was sufficiently small, i.e. $a_{0}(0)=1$ and $b_{m}(0)=0(m=0,1,2, \cdots, K)$ and then demonstrated over the wide range of the PWGCC parameters as the behavior of the mode coupling while on the fundamental level, without letting higher-order modes complicate the matter. For the coupling of the composite side-polished fiber and PWGCC, a pair of hypothetical walls were also imposed at a significant distance $y= \pm D$ from the origin of the coordinates, where $D$ is a constant. The transverse slab mode $T_{n}(n=0,1,2, \cdots, K)$ approaches zero when $y$ reaches $\pm D$. This model has already been applied to a distributed multimode-mode PWGCC coupler. Only the case of the $T E$ mode was analyzed based on satisfying the boundary condition on the surface with the PEC and a pair of hypothetical walls. Further denotions include $\beta_{s n}(n=0,1,2, \cdots, K)$ as $\beta_{s n}^{2}=\beta_{s}^{2}-\sigma_{n}^{2}$ and $\sigma_{n}=\pi(n+1 / 2) / D,(n=0,1,2, \cdots, K)$, where $\beta_{s}$ is the maximum propagation constant of the set of slab modes traveling along the $z$ axis. $D=500 \mu \mathrm{m}$ is used as the distance between each hypothetical wall. Fig. 2(a) indicates that, when $n_{f}>n_{s}$, the coupling between each structure was ineffective, only a fraction of the launched power in the fiber fluctuated between the fiber and the PWGCC, and the light power remained largely in the fiber. When $\beta_{f 0}>\beta_{s}$ and in the absence of coupling, the fiber mode was not in phase synchronism with any PWGCC modes and very little power was coupled from the fiber to the PWGCC. Fig. 2(b) shows the relationship between the value of $n_{f}$ relative to that of $n_{s}$. Lowering the value $n_{f}$ generally caused the power oscillation to decay slightly and increased the rate of decay of the power beating, thereby transferring power from the fiber to the PWGCC due to the reduced phase mismatch. When the value of $n_{f}$ was equal that of $n_{s}$, the amount power coupled out of the fiber was also strongly dependent. Finally, when the value of $n_{f}$ was less than that of $n_{s}$, the fiber mode decayed in such a way that the smaller the vaue of $n_{f}$ relative to the value of $n_{s}$, the stronger the coupling to the PWGCC, as shown in Fig. 2(d). Thus, the power oscillated with a high strength and died out. Since the rate of decay of the power beating and power was substantially from the fiber to the PWGCC, the absolute square of the fiber mode amplitude $\left|a_{0}\right|^{2}$ reduced to nearly zero beyond a certain propagation distance (typically $2000 \mu \mathrm{m}$ ) from the initial value of $\left|a_{0}\right|^{2}$ at $z=0$. Fig. 2(e) shows the exponential curve fit of $\left|a_{0}\right|^{2}=e^{-\alpha z}$ at various $s$, where $\alpha=K_{f 0 m} K_{s m 0} \frac{\beta_{f 0}}{\sigma_{m}} D$ is the attenuation coefficient of the fiber mode due to radiation loss from the PWGCC, when $\beta_{s m}=\beta_{f 0}, \sigma_{m}=(2 m+1) \pi /(2 D)$, and $m=0,1,2, \cdots, K$. As shown in Fig. 2(e), the power beating and power transferred to the PWGCC was larger as the distance between the structures became smaller. The light transfer between the fiber and the PWGCC was found to depend strongly on the relationship between their propagation constants $\beta_{f 0}$ and $\beta_{s}$. When $\beta_{s}$ was considerably larger than $\beta_{f 0}$, the PWGCC also acted as a sink for the optical power in the fiber, so that the total power launched initially into the fiber was spread out laterally in the PWGCC. A compound equation was also used to represent the transverse electric field for the propagating direction at $y=0$ and Newton-Raphson's method used to obtain the eigenvalue from the compound Eqs. (18) and (19). These modes exhibited a common propagation constant for the entire system and a stationary power distribution beyond a certain distance of propagation. When the PWGCC was located at $s=4 \mu \mathrm{m}$ from the side-polished fiber and the refractive index of the fiber was $n_{f}>n_{s}$, Fig. 3(a) illustrates that most of the compound-mode field remained within the vicinity of the fiber as opposed to the PWGCC. However, there were some deformations of the symmetric mode of the isolated fiber due to minimal coupling with the PWGCC. As the value of $n_{f}$ approached yet remained lower than $n_{s}$, as shown in Figs. 3(b), (c), and (d), large humps appeared in the field around the position of the PWGCC and the variation of the refractive index $n_{f}$ caused more deformation of the pure fiber mode. The large fractions of mode power were gradually transferred to the PWGCC from the fiber. When the second compound-mode eigenvalue from Eqs. (18) and (19) was used, Fig. 3(e) shows that the nearly all of the mode power was transferred to the PWGCC when $s=3 \mu \mathrm{m}$. As shown in Figs. 4(a), (b), (c), and (d), a decrease in the value of $n_{f}$ generally caused the amplitude of the field to increase in the vicinity of the fiber. Therefore, the smaller the distance between the side-polished fiber and the PWGCC, the higher the amplitude of the electric field. Figs. 4(a), (b), (c), and (d) also indicate that these dominant compound modes were indeed ridge modes, as the fields at the center of the PWGCC were concentrated in the vicinity of the fiber. The peak values of the ridgemode 


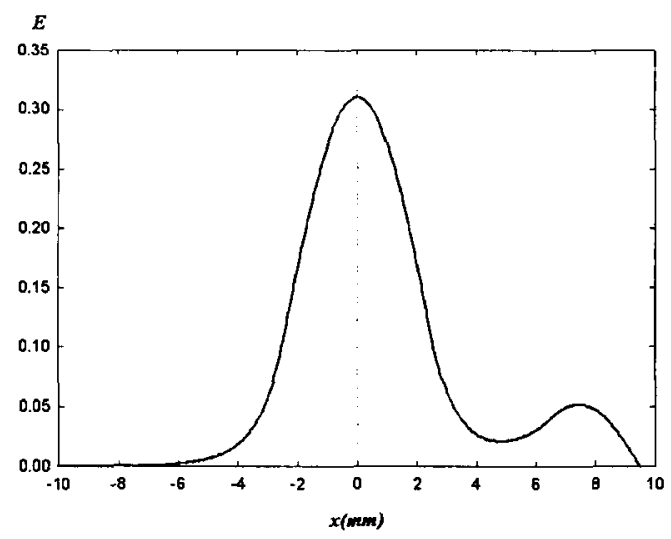

(a)

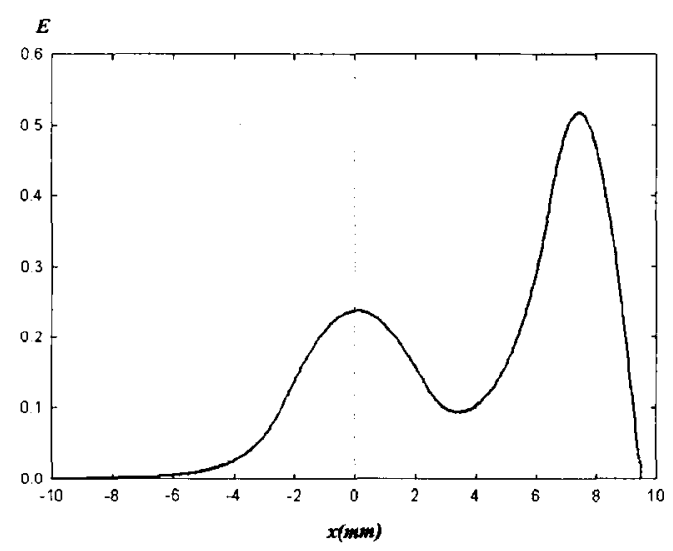

(c)

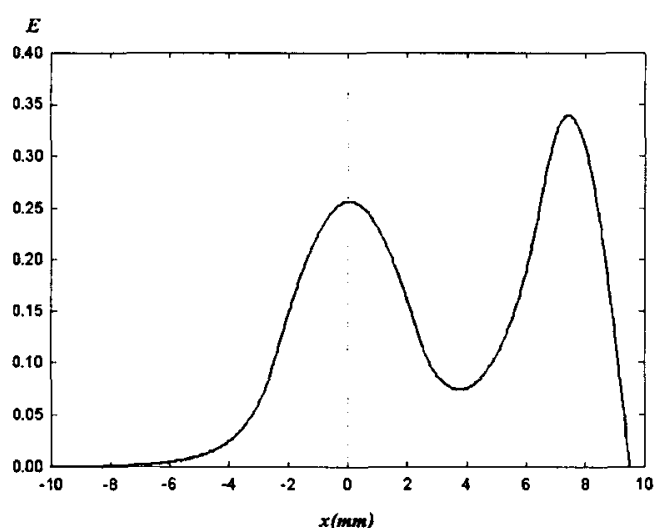

(b)

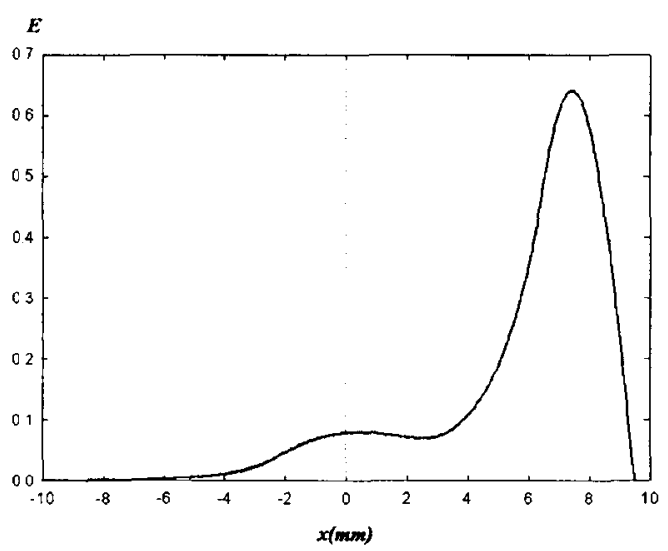

(d)

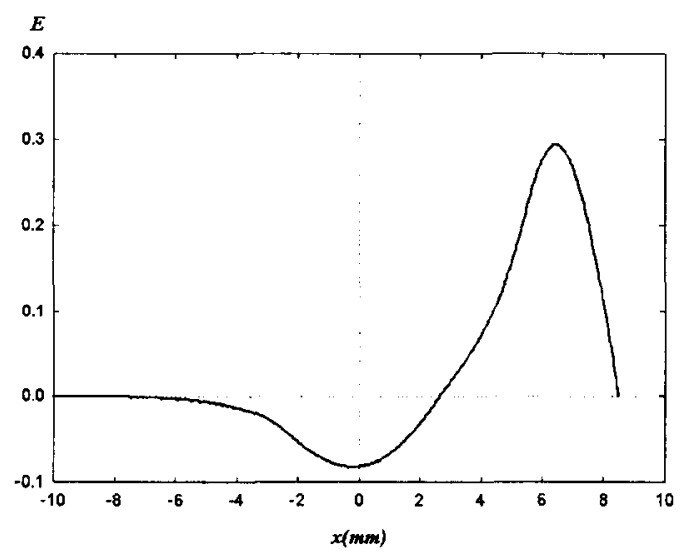

(e)

FIG. 3. Fields $E$ of dominant compound eigenmodes of fiber-PWGCC as function of $x$ in plane $y=0 . n_{f}=($ a) 1.4817 , (b) 1.4756 , (c) 1.4745 and (d) 1.4709 , (e) field $E$ of second ridge-modes for $n_{f}=1.4817$ as function of $x$ in plane $y=0$.

fields were dependent on the relative values of $n_{f}$, and the rate of the power transfer to the PWGCC was generally slightly higher. Fig. 4(e) shows the confinement of the fields around the center of the fiber, even though most of the light energy was concentrated in the plane of the PWGCC with the second largest compound mode at $s=3 \mu \mathrm{m}$. According to Figs. 2, 3, and 4 , the simulation results matched very well with the physical phenomena reported in references [6] and $[7]$. 


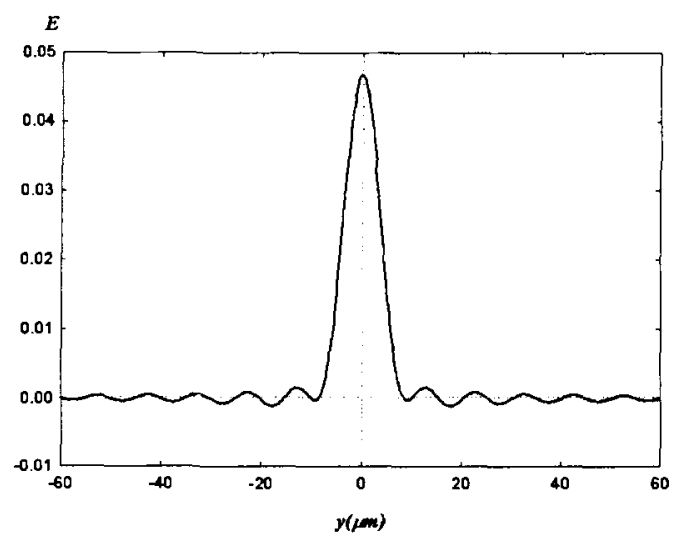

(a)

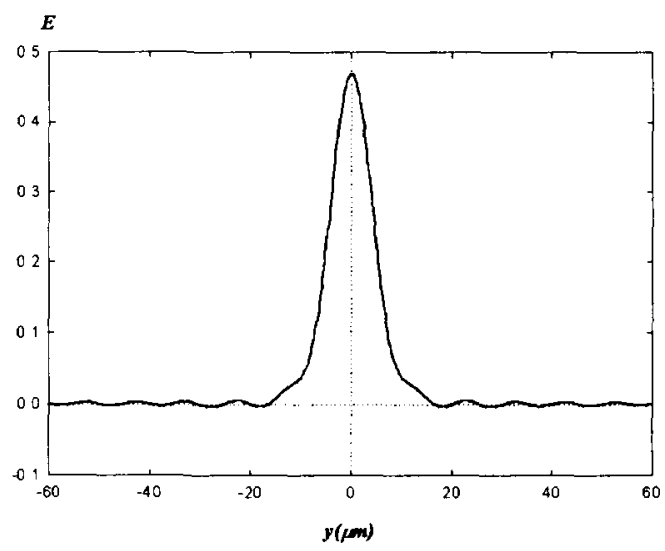

(c)

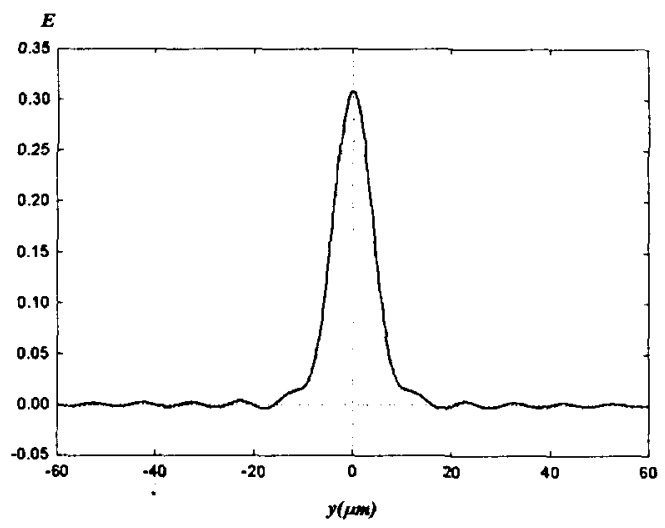

(b)

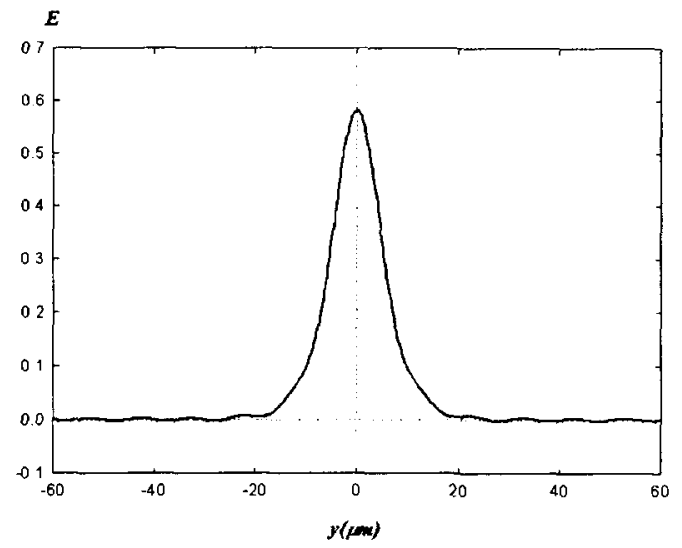

(d)

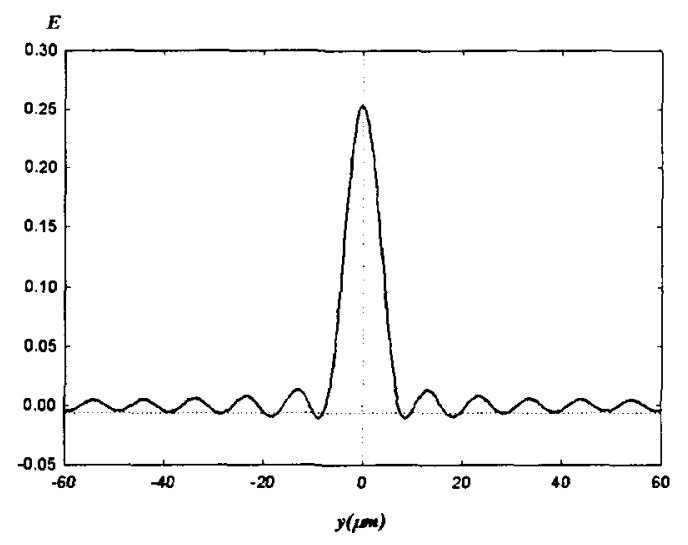

(e)

FIG. 4. Fields $E$ of dominant compound eigenmodes of fiber-PWGCC as function of $y$ in plane $x=7 \mu \mathrm{m}$. $n_{f}=$ (a) 1.4817 , (b) 1.4756 , (c) 1.4745 and (d) 1.4709 , (e) field $E$ of second ridge-modes for $n_{f}=1.4817$ as function of $y$ in plane $x=8 \mu \mathrm{m}$.

\section{CONCLUSION}

In conclusion, an analysis of an asymmetric composite fiber-PWGCC was conducted and the drain of optical power from the fiber to the PWGCC was shown to depend on a combination of the relative values of $n_{f}$ and $n_{s}$. The properties of the coupling between the side-polished fiber and PWGCC for the ridge modes

were presented based on a CMT model, while taking account of the multimode PWGCC. The exchange of energy between the fiber and the PWGCC was visualized by comparing the light intensity at different cross sections of the fiber-slab combination along the propagation direction and the characteristics of the fiber 
and PWGCC mode-coupling presented, especially the features of the power oscillations and beatings. As shown in each figure, when $n_{s}$ was lower than $n_{f}$, the light was generally well confined within the fiber core. However, as soon as $n_{s}$ approached or became higher than $n_{f}$, the mode beating, decay, and amount of the power transferred could be readily tuned by a small variation in the value $n_{f}$. Finally, based on the coupler presented in the current study, the ability to achieve the desired light coupling and power transfer performance would appear to be feasible by optimizing the combination of the structural parameters between the side-polished fiber and the PWGCC. Accordingly, this device has many potential applications in different fields.

\section{APPENDIX A: COUPLING COEFFICIENT}

The coupling coefficients computed from (13)-(15) are exact, except for (15) listed below. A largeargument asymptotic approximation of the modified Bessel function $K_{0}$ was used in (15).

$$
\begin{gathered}
Q_{f 00}=\frac{\pi V^{2} N_{f}^{2}}{4 \sqrt{2} \gamma_{f}^{2} t^{2} \beta_{f 0} K_{0}^{2}\left(\gamma_{f} a\right)} \cdot\left\{\operatorname{erf}\left[2 \gamma_{f}(a+s+t)\right]-\operatorname{erf}\left[2 \gamma_{f}(a+s)\right]\right\} \\
Q_{s m n}=\frac{\pi N_{s}^{2} V_{f}^{2}}{2 \beta_{s m}} \cdot \exp \left[-2 \gamma_{s}(a+s+t)\right] \cdot\left\{\frac{I_{1}\left[\sqrt{4 \gamma_{s}^{2}-\left(\sigma_{m}+\sigma_{n}\right)^{2}} a\right]}{\sqrt{4 \gamma_{s}^{2}-\left(\sigma_{m}+\sigma_{n}\right)^{2}} a}+\frac{I_{1}\left[\sqrt{4 \gamma_{s}^{2}-\left(\sigma_{m}-\sigma_{n}\right)^{2}} a\right]}{\sqrt{4 \gamma_{s}^{2}-\left(\sigma_{m}-\sigma_{n}\right)^{2}} a}\right\} \\
K_{f 0 n}=\frac{\pi N_{f} N_{s} V_{f}^{2}}{\beta_{f 0} a J_{0}\left(k_{f} a\right)} \cdot \frac{\exp \left[-\gamma_{s}(a+s+t)\right]}{k_{f}^{2}+\gamma_{s}^{2}-\sigma_{n}^{2}} \cdot\left\{\sqrt{\gamma_{s}^{2}-\sigma_{n}^{2}} \cdot J_{0}\left(k_{f} a\right) \cdot I_{1}\left(\sqrt{\gamma_{s}^{2}-\sigma_{n}^{2}} a\right)+k_{f} J_{1}\left(k_{f} a\right) \cdot I_{0}\left(\sqrt{\gamma_{s}^{2}-\sigma_{n}^{2}} a\right)\right\}( \\
K_{s m 0}=\frac{\pi V^{2} N_{f} N_{s} \cdot \exp \left[-(a+s) \sqrt{\gamma_{f}^{2}+\sigma_{n}^{2}}-\gamma_{s} t\right]}{2 \beta_{s n} t^{3} K_{0}\left(\gamma_{f} a\right)} \cdot \frac{t\left(\gamma_{s}+\sqrt{\gamma_{f}^{2}+\sigma_{n}^{2}}\right)-V \cdot \exp \left[-t \sqrt{\gamma_{f}^{2}+\sigma_{n}^{2}}\right]}{\left(k_{s}^{2}+\gamma_{f}^{2}+\sigma_{n}^{2}\right) \sqrt{\gamma_{f}^{2}+\sigma_{n}^{2}}}
\end{gathered}
$$

Where $V^{2}=\left(n_{s}^{2}-n_{c}^{2}\right) k^{2} t^{2} . J_{n}$ and $I_{n}$ are the Bessel and modified Bessel functions of order $n, K_{0}$ is the zero-order modified Hankel function, and $\operatorname{erf}(x)$ is the error function.

*Corresponding author : tree@palgong.knu.ac.kr.

\section{REFERENCES}

[1] C. A. Millar, M. C. Brierley, and S. R. Mallinson, Opt. Lett. 12, 284 (1987).

[2] W. Johnstone, G. Stewart, B. Culshaw, and T. Hart, Electron. Lett. 24, 866 (1988).
[3] W. Johnstone, S. Murray, G. Thursby, M. Gill, A. McDonach, D. Moodie, and B. Culshaw, Electron. Lett. 27, 894 (1991).

[4] G. Fawcett, W. Johnstone, I. Andonovic, D. J. Bone, G. Harvey, N. Carter, and T. G. Ryan, Electron. Lett. 28, 985 (1992).

[5] G. Thursby, W. Johnstone, K. McCallion, D. Moodie, and B. Culshaw, in Proc. 8th Optical Fiber Sensors Conf., Monterey, CA, Jan. 29-31, 1992, pp. 197-200.

[6] D. Marcuse, J. Lightwave Technol. 7, 122 (1989).

[7] S. Zheng, L. N. Binh, and G. P. Simon, J. Lightwave Technol. 13, 244 (1995).

[8] E. A. J. Marcatili, Bell Syst. Tech. J. 53645 (1974).

[9] D. Gloge, Appl. Opt. 10, 2252 (1971).

[10] A. Ishimaru, Electromagnetic wave propagation, radiation, and scattering (Englewood Cliffs, N.J.: Prentice-Hall Inc., 1991). 